\title{
Conceito e Relevância dos Interesses Profissionais no Desenvolvimento de Carreira: Estudo Teórico
}

\author{
Karen Cristina Alves Lamas ${ }^{1}$ \\ Universidade Salgado de Oliveira, Juiz de Fora, MG, Brasil
}

\begin{abstract}
Resumo
Os interesses profissionais vêm sendo pesquisados desde o início do século XX. Ao longo desse tempo várias teorias sobre o desenvolvimento de carreira abordaram a importância de sua avaliação. O objetivo do presente artigo é apresentar definições conceituais e processos desenvolvimentais dos interesses de acordo com quatro teorias amplamente divulgadas, inclusive no contexto brasileiro. Assim, a partir da revisão da Teoria das Personalidades Vocacionais, da Teoria Desenvolvimentista, da Teoria Sociocognitiva do Desenvolvimento de Carreira e da Teoria Construtivista de Savickas foi verificado que não há grandes divergências entre as definições conceituais. Porém, a literatura atual apoia o conceito de traço ou disposição relativamente estável para responder a estímulos do ambiente. Quanto às hipóteses que fundamentam a formulação dos interesses há diferenças de ênfase em algumas variáveis, por exemplo, experiências de aprendizagem e condições socioculturais. Ressalta-se a necessidade de novas pesquisas, principalmente no contexto nacional, que possuam delineamento longitudinal, busquem contextualizar as variáveis envolvidas no processo de desenvolvimento, e construir instrumentos de avaliação que possam ser utilizados em contextos educacionais e organizacionais.
\end{abstract}

Palavras-chave: Desenvolvimento profissional, interesses profissionais, escolha profissional, revisão de literatura.

\section{Concept and Relevance of Vocational Interests in Career Development: A Theoretical Study}

\begin{abstract}
Vocational interests have been researched since the early twentieth century. During that time several theories about career development addressed the importance of their evaluation. The aim of this paper is to present conceptual definitions and developmental processes of interests according to four theories widely publicized, including in the Brazilian context. Thus, from the review of the Theory of Vocational Personalities, the Developmental Theory, the Socio-Cognitive Theory of Career Development and Constructivist Theory of Savickas was found that there is no conceptual oppositions between the definitions. But the current literature supports the concept of trait or disposition relatively stable to respond to environmental stimuli. Regarding the assumptions underlying the formulation of interests there are emphasis differences in some variables, for example, learning experiences and sociocultural conditions. It emphasizes the need for further research, especially in the national context, that has a longitudinal
\end{abstract}

Endereço para correspondência: Universidade Salgado de Oliveira - UNIVERSO, Av. dos Andradas, 731, Morro da Glória, Juiz de Fora, MG, Brasil 36036-000. E-mail: karen_lammas@yahoo.com.br Trabalho derivado da tese de doutorado da autora, desenvolvida com apoio da Fundação de Amparo à Pesquisa do Estado de São Paulo - FAPESP e da Coordenação de Aperfeiçoamento de Pessoal de Nível Superior CAPES. 
design, seeks to contextualize the variables involved in the development process, and build assessment tools that can be used in educational and organizational contexts.

Keywords: Professional development, professional interests, career choice, literature review.

\section{Concepto y Relevancia de los Intereses Profesionales en el Desarrollo Profesional: Estudio Teórico}

\section{Resumen}

Los intereses profesionales se han estudiado desde principios del siglo XX. Durante ese tiempo varias teorías sobre el desarrollo profesional abordaron la importancia de la evaluación. El propósito de este trabajo es presentar los conceptos y los procesos de desarrollo de los intereses de acuerdo a cuatro teorías ampliamente publicitados, incluso en el contexto brasileño. Así, a partir de la revisión de la Teoría de Personalidades Profesional, la Teoría del Desarrollo, la Teoría Socio-Cognitiva de Desarrollo Profesional y Teoría Constructivista del Savickas no hubo grandes diferencias entre las definiciones conceptuales. Sin embargo, la literatura actual apoya el concepto de rasgo o disposición relativamente estable para responder a los estímulos ambientales. Con respecto a los supuestos que subyacen en la formación de los intereses, diferencias de énfasis sobre algunas variables se verificaron, por ejemplo, las experiencias de aprendizaje y las condiciones sociales y culturales. Se hace hincapié en la necesidad de una mayor investigación, especialmente a nivel nacional, que buscan un diseño longitudinal, contextualizar las variables que intervienen en el proceso de desarrollo, y construir instrumentos de evaluación que se pueden utilizar en contextos educativos y organizativos.

Palabras clave: Desarrollo profesional, intereses profesionales, escogimiento profesional, revisión de literatura.

No ano de 2013 a National Career Development Association completou cem anos de existência, e para celebrar foi construída uma monografia com os principais conceitos e práticas que alavancaram o campo do desenvolvimento de carreira neste centenário. Dentre essas concepções, tais como adaptabilidade de carreira, orientação vocacional, justiça social e autoeficácia profissional, foi inserido o inventário de interesses. Para Savickas (2013), o inventário de interesses está entre as dez ideias vitais da área, pois a avaliação dos interesses profissionais contribui em processos de intervenção que visem ao desenvolvimento humano em períodos de transição ao longo da vida. Além disso, é tema recorrente na literatura científica (Leitão \& Miguel, 2001; Sodano, 2015). Assim, considera-se importante disponibilizar uma revisão do conceito de interesses para aqueles que se preocupam com pesquisa e/ou avaliação desse construto psicológico.
O início dos estudos sobre interesses profissionais data da primeira metade do século XX (p.ex., Fryer, 1931) e foram realizados principalmente com a finalidade de construir inventários, tais como o Strong Vocational Interest Blank (SVIB) em 1928 e o Kuder Preference Record-Vocational em 1938 (Harrington \& Long, 2013; Mattiazzi, 1977). Embora vários autores tenham buscado, ao longo dos anos, conceituar interesses profissionais e explicar seu processo de desenvolvimento, os trabalhos foram desenvolvidos desarticuladamente e focalizados em dados empíricos, de forma que não deixaram transparecer a evolução do conceito (Leitão \& Miguel, 2001; Savickas, 1999).

Para compreender a conceptualização de interesses alinhada com essa abordagem empirista, serão apresentadas contribuições teóricas sobre o construto que permitem sua avaliação por meio de inventários ou instrumentos de autorrelato (Werlang, Villemor-Amaral, \& 
Nascimento, 2010). Tais teorias são bastante utilizadas em processos de intervenção e investigadas no contexto brasileiro e internacional. Especificamente, são analisadas quatro abordagens teóricas, a saber, a teoria das personalidades vocacionais (Holland, 1997), a teoria sociocognitiva do desenvolvimento de carreira (Lent, Brown, \& Hackett, 1994), a desenvolvimentista de Super (1980) e a construtivista de Savickas (1995).

A teoria das personalidades vocacionais de Holland (1997) e a teoria sociocognitiva (Lent et al., 1994) oferecem hipóteses específicas sobre os interesses profissionais que são amplamente investigadas por estudos empíricos. De modo distinto, a teoria desenvolvimentista de Super (1980) e a teoria construtivista de Savickas (1995) embora tenham realizado contribuições conceituais importantes sobre os interesses profissionais, esse construto é secundário diante de outros (p.ex., saliência da carreira, maturidade vocacional, adaptabilidade) que compõem seus respectivos modelos teóricos. Portanto, no que se referem aos interesses, suas contribuições são designadamente de conceptualização e aplicação prática dentro de modelos de orientação profissional, fato que justifica o pequeno número de pesquisas empíricas que investigaram os interesses profissionais tendo como base essas perspectivas. A seguir são apresentadas as asserções sobre os interesses profissionais propostas em cada teoria e citados alguns estudos empíricos que ilustrem a aplicação.

\section{Teoria das Personalidades Vocacionais e Ambientes de Trabalho}

Uma das teorias mais tradicionais sobre a escolha das profissões que aborda os interesses foi desenvolvida por John Holland (1959, 1996, 1997) e teve suas primeiras publicações divulgadas no final dos anos 1950. Salienta-se que nesta seção será utilizado, principalmente, o texto publicado em 1997, além das outras referências do autor.

Holland construiu sua teoria com base na experiência que possuía como conselheiro vocacional, na literatura sobre o assunto e no desenvolvimento de um primeiro instrumento de mensuração denominado Vocational Preference Inventory publicado em 1958. Esse autor define os interesses profissionais como uma forma de expressão das características de personalidade no mundo do trabalho. A partir de sua prática, Holland elaborou um modelo tipológico para explicar a escolha profissional que relaciona seis tipos de personalidades a seis modelos de ambientes profissionais, classificados sob a mesma terminologia. Portanto, o modelo é conhecido pela sigla RIASEC, que se refere aos tipos Realista, Investigativo, Artístico, Social, Empreendedor e Convencional. Os tipos podem ser dispostos, nessa ordem, nos vértices de um hexágono, de forma que, quanto mais próximos, mais semelhanças existem entre eles. Por isso, a perspectiva é também chamada de modelo hexagonal.

Pessoas com características do tipo Realista gostam de trabalhos manuais, práticos e técnicos, que necessitam de ferramentas, máquinas ou equipamentos eletrônicos e tendem a evitar ambientes que exijam interação social. $\mathrm{O}$ perfil Investigativo está relacionado à preferência por atividades acadêmicas e de pesquisa, que exijam certo nível de curiosidade e capacidade analítica, evitando situações que demandam persuadir os outros. O tipo Artístico abrange pessoas que se reconhecem como introspectivas, intuitivas e originais, que gostam de se expressar por meio de atividades artísticas (p.ex., literárias e musicais) e se afastam da rotina e de ambientes convencionais (Holland, 1996,1997).

Os indivíduos do tipo Social gostam de ajudar e compreender os outros, percebem-se com capacidade para exercer atividades de ensino e aconselhamento e evitam tarefas mecânicas ou técnicas. O perfil Empreendedor envolve tarefas em que há a necessidade de convencer pessoas para atingir objetivos organizacionais ou econômicos. Indivíduos com esse perfil apresentam capacidade de liderança e persuasão, e podem não possuir competências para atuar em ambientes de pesquisa. Pessoas do tipo Convencional gostam de sistematizar, organizar e manipular dados e informações, também, percebem-se com habilidades empresariais e pouca capacidade artística (Holland, 1996,1997). 
Segundo Holland (1996, 1997), a congruência entre personalidade e ambiente ocupacional está diretamente relacionada à satisfação no trabalho, ao nível de desempenho e à estabilidade profissional. Os modelos de ambientes profissionais podem ser identificados pelas exigências que o meio requer dos colaboradores e pela prevalência de determinado tipo de personalidade, assim, se há um grande número de funcionários do tipo Realista, provavelmente aquele ambiente possui características realistas. Já os tipos de personalidades são produtos de interações específicas entre fatores pessoais e socioculturais, tais como a convivência com os pares, hereditariedade, atitude dos pais, classe econômica, ambiente físico e social.

Para explicar sua concepção de desenvolvimento, o autor propõe que, ainda na infância, fatores biológicos e experiências do meio sociocultural contribuem para a formação de preferência por determinadas atividades e aversão a outras. Posteriormente, essas preferências vão se transformando em interesses bem definidos de acordo com a percepção de satisfação pessoal e recompensas. A busca por tais interesses leva ao desenvolvimento de algumas competências mais especializadas e à negligência de outras em potencial. Ao longo do tempo, ocorre uma diferenciação dos interesses, acompanhada pela solidificação de valores. Esse conjunto de eventos dá origem a diferentes tipos de interesses ou personalidades vocacionais. Cada um possui predisposição a exibir um repertório comportamental específico e são compostos por uma combinação de preferências e rejeições, habilidades, autoconceito, valores e estilos de enfrentamento.

Uma vez que os interesses configuram a expressão da personalidade no contexto ocupacional e, ainda, como afirma Holland (1996, 1997), os inventários de interesses podem ser vistos como inventários de personalidade, verifica-se ao longo de seus textos uma equivalência na utilização desses dois conceitos. Portanto, fala-se em tipos de interesses ou de personalidades vocacionais. Os tipos são parâmetros idealizados pelos quais os indivíduos podem ser mensurados e comparados a fim de verificar uma aproximação ou semelhança, de forma que as pessoas não detêm características de apenas um; os indivíduos apresentam um padrão ou perfil de personalidade em que um ou dois tipos de interesses/ personalidade são mais proeminentes. Para avaliação dos tipos, o autor construiu um instrumento denominado Self-Directed Search - SDS, que foi traduzido e validado para vários países, inclusive para o Brasil (Primi, Muniz, Mansão, \& Nunes, 2010).

Os interesses podem ser mensurados desde a infância e, principalmente, usados para predizer escolhas profissionais posteriores (Holland, 1996). Para contribuir com a avaliação e mensurar o poder preditivo desta, Holland (1997) desenvolveu os conceitos secundários, denominados nitidez ou diferenciação, consistência, congruência e identidade.

A nitidez ou diferenciação se refere à clareza do perfil de interesses, ou seja, dentre as dimensões ou tipos avaliados verifica-se a distância entre o maior e o menor escore, quanto maior esse valor, ou seja, a nitidez, mais poder preditivo tem os interesses. A consistência diz respeito ao nível de semelhança, indicado pelo modelo hexagonal, entre os tipos mais proeminentes resultantes da avaliação. Quanto mais próximos estiverem os tipos, mais estável é o comportamento vocacional do indivíduo. Já a congruência é a equivalência entre o tipo e o ambiente. Por exemplo, espera-se que uma pessoa Social trabalhe em um ambiente também Social, pois nele encontrará as oportunidades e as recompensas que necessita. Portanto, esse índice indica a persistência do indivíduo em uma ocupação. Por fim, o conceito de identidade sugere o quanto o perfil de interesses está claro, estável e de acordo com os objetivos e as habilidades do sujeito.

Segundo Nauta (2010), a teoria de Holland tem a proficuidade de ser facilmente testada e aplicada. De acordo com a revisão de literatura realizada pela autora, as pesquisas empíricas corroboram a existência dos seis tipos RIASEC para pessoas e ambientes, sendo que o modelo já foi reaplicado em muitos países e adotado como fundamentação para diversos instrumentos de avaliação dos interesses profissionais.

Quanto aos conceitos secundários, a autora verificou que há apoio para algumas hipóteses 
e necessidade de mais investigação para outras. Para as proposições relacionadas à congruência há suporte empírico para sua associação com estabilidade profissional, satisfação com a carreira e persistência, contudo o tamanho do efeito tende a ser pequeno. Para consistência e diferenciação, o poder explicativo tem sido menor do que o esperado em relação a essas variáveis, enquanto para identidade, os resultados dos diferentes estudos têm sido contraditórios. Contudo, Nauta (2010) alega que são poucas as pesquisas realizadas para a investigação desses três últimos conceitos.

Com o objetivo de provar a utilidade das hipóteses de consistência e diferenciação, Tracey, Wille, Durr e De Fruyt (2014) adotaram um novo método de operacionalização desses conceitos, no qual é utilizado todo o perfil RIASEC e as diferenças entre as pontuações em cada tipo, utilizando o ajuste do cosseno e a amplitude. Assim, os autores comprovaram que além de relações diretas, esses conceitos também possuem um efeito moderador importante entre congruência e resultados de carreira, como satisfação e estabilidade ocupacional. Outros estudos que investigaram os tipos e os conceitos secundários podem ser visualizados em Hirschi $(2010,2011)$ e Huang e Pearce (2013).

Salienta-se que o modelo tipológico de Holland (1959, 1996, 1997) é indicado para a classificação dos interesses profissionais por diversos autores (Lent \& Brown, 2006a; Savickas, 2005; Super \& Bohn, 1970/1980; dentre outros) que construíram teorias sobre o desenvolvimento ocupacional, mas não apresentaram um instrumento específico para a avaliação. A indicação do modelo RIASEC pelos referidos autores ocorre embora deixem claro que a concepção de desenvolvimento dos interesses é distinta dos pressupostos pela teoria das personalidades vocacionais de Holland.

\section{Teoria Sociocognitiva do Desenvolvimento de Carreira}

Uma das abordagens elaboradas mais recentemente que inclui o desenvolvimento dos interesses profissionais é a Teoria Sociocognitiva do Desenvolvimento de Carreira. Apresentada por
Lent et al., em 1994, essa perspectiva derivada da Teoria Social Cognitiva de Bandura (1977, 1982) reúne os conceitos mais relevantes para a compreensão do desenvolvimento profissional e acadêmico, especificamente no que se refere aos interesses, às escolhas e ao desempenho nas atividades ocupacionais e educacionais. Posteriormente, a teoria foi ampliada para abranger a satisfação/bem-estar em contextos profissionais e acadêmicos (Lent \& Brown, 2006b, 2008) e a autogestão da carreira (Lent \& Brown, 2013). Assevera-se que são destacados aqui os pontos teóricos e empíricos que explanam sobre os interesses profissionais.

Lent et al. (1994) compreendem os interesses como padrões de gostos, aversões e indiferenças acerca de atividades e funções ligadas a uma profissão. Para explicar o desenvolvimento dos interesses profissionais, os autores elaboraram um modelo, no qual a autoeficácia e as expectativas de resultado conduzem à formação dos interesses, sendo tais crenças derivadas da combinação de variáveis pessoais (p. ex., origem étnico-racial, sexo e predisposições hereditárias), contextuais (p. ex. oportunidades educacionais, modelos, suporte social e financeiro, influências familiares) e experiências de aprendizagem.

As crenças de autoeficácia se referem ao quanto uma pessoa se considera capaz de realizar bem uma determinada tarefa (Bandura, 1977). Esse é um construto que possui domínios específicos, ou seja, o mesmo indivíduo possui diferentes níveis de autoeficácia de acordo com distintas atividades. Tais crenças são construídas ao longo das experiências de aprendizagem e possuem quatro fontes principais: experiência de êxito, aprendizagem vicária, persuasão verbal e indicadores fisiológicos. Esses termos foram traduzidos por Nunes (2007), mais informações sobre as definições estão disponíveis em Bandura $(1977,1982)$ e Pajares e Olaz (2008).

Geralmente, as crenças de autoeficácia predizem as expectativas de resultado, que se referem às perspectivas de recompensa ao emitir determinado comportamento (Bandura, 1977). Essa antecipação de resultados é desenvolvida, também, pelas experiências de aprendizagem pessoal e vicária, interação com pessoas mais 
próximas, instituições religiosas e culturais e pela mídia em geral (Lent et al., 1994).

Uma vez que os indivíduos tendem a preferir tarefas em que há percepção de competência e confiança - autoeficácia, bem como atividades nas quais antecipam resultados positivos - expectativas de resultado (Bandura, 1977; Pajares \& Olaz, 2008), fica evidente a contribuição desses conceitos para o desenvolvimento dos interesses profissionais (Lent et al., 1994). Por exemplo, a experiência em desenvolvimento de software ou jogos de videogames pode levar ao interesse pelo curso de ciência da computação (Lent \& Brown, 2006a).

Consequentemente, os interesses emergentes combinados com as crenças de autoeficácia e expectativas de resultado conduzem à formulação e revisão de objetivos ${ }^{2}$ relacionados à profissão (Lent et al.,1994; Lent, Hackett, \& Brown, 2004). Essas intenções de escolha podem ser alteradas por diversos fatores, como os financeiros ou educacionais, pois, nos momentos próximos à escolha, as condições contextuais filtradas pelos fatores pessoais constituem influências que exercerão papel fundamental, configurando-se como fonte de apoio ou obstáculo para a tomada de decisão, atuando como moderadores entre interesse-objetivos e objetivos-ações (Lent et al., 1994). Por isso, os objetivos são mais consistentes quando podem ser implementados num futuro próximo (Lent \& Brown, 2006a).

É importante lembrar que a Teoria Sociocognitiva do Desenvolvimento de Carreira (Lent et al., 1994; Lent et al., 2004) deriva da teoria de Bandura (1977), assim seus fundamentos são embasados no pressuposto do determinismo recíproco, isto é, fatores pessoais, comportamentais e ambientais mantêm uma relação triádica na produção das ações humanas. Dessa forma, o indivíduo tem a oportunidade de ser agente da sua própria trajetória de desenvolvimento, por meio da reflexão sobre suas crenças, interesses e cursos de ação (Bandura, 1978/2008). Embo-

2 De acordo com outros trabalhos publicados no contexto nacional (Ambiel \& Noronha, 2014; Nunes, 2009), o termo 'goals' foi traduzido como 'objetivos' e não como 'metas'. ra as hipóteses propostas por Lent et al. (1994) sugiram uma direção de influência entre as variáveis, os autores admitem a possibilidade de um relacionamento bidirecional ou de alguma variável ter mais peso que a outra em circunstâncias específicas.

Por conseguinte, Lent et al. (1994; Lent et al., 2004) argumentam que para alguns indivíduos, os interesses nem sempre irão predizer a escolha profissional, de forma que as crenças de autoeficácia ou as expectativas de resultados podem ter mais impacto no momento da decisão quando as oportunidades para a implementação dos interesses são percebidas como limitadas. Acrescentam que a escolha de objetivos e a revisão das crenças podem acontecer várias vezes ao longo da vida, principalmente quando o desempenho na atividade escolhida não ocorre de maneira satisfatória. Desta compreensão decorre a ideia de que os interesses não são estáticos, embora sejam necessários eventos de ampla significância para modificar as crenças subjacentes a sua formação. Em um curso de desenvolvimento ideal, no final da adolescência ou início da idade adulta, os interesses estão se estabilizando e, ao longo do tempo, tendem a se tornar mais consistentes (Lent et al., 1994).

Ao avaliar interesses é preciso considerar o nível de especificidade desejado, de forma que a avaliação pode apresentar atividades que em conjunto apontem uma profissão a ser seguida ou indicar campos e áreas de conhecimento. A avaliação pode ser ainda mais específica, por exemplo, ao examinar o interesse de estudantes de psicologia pelas atividades de pesquisa ou de clínica. Os autores salientam que os instrumentos devem ser adequados às características do cliente (p.ex., idade e experiências de vida), além da redação dos itens ser atual ao contexto social (Lent \& Brown, 2006a).

Diversas pesquisas vêm sendo realizadas para investigar as hipóteses propostas pela Teoria Sociocognitiva do Desenvolvimento de Carreira (Lent et al., 1994; Lent et al., 2004) no contexto nacional (p.ex., Nunes \& Noronha, 2009, 2011) e internacional (p.ex., Byars-Winston, Estrada, Howard, Davis, \& Zalapa, 2010; Tracey, 2010). Ao realizar uma meta-análise, Sheu et al. 
(2010) tiveram como objetivo sintetizar dados relevantes para testar as hipóteses de formação dos interesses e da escolha profissional propostas pela Teoria Sociocognitiva do Desenvolvimento de Carreira (Lent et al., 1994), utilizando estudos que investigaram as variáveis por meio de dimensões baseadas nos tipos profissionais de Holland (1997). A partir dos dados disponíveis em 40 artigos, foi possível testar duas versões do modelo sociocognitivo.

A primeira versão incluiu quatro variáveis, quais sejam, autoeficácia, expectativas de resultado, interesses e objetivos de escolha com amostras que investigaram os tipos Artístico, Social e Convencional. A segunda analisou, além das variáveis anteriores, o apoio e as barreiras para a escolha, completando seis variáveis, em amostras com os tipos Realistas, Investigativo e Empreendedor. Os resultados foram em sua maior parte consistentes com as expectativas teóricas. O modelo com seis variáveis foi o que indicou um suporte melhor ao representar a relação entre condições contextuais e objetivos como sendo parcialmente mediada pela autoeficácia e expectativas de resultado, ao invés de gerar apenas ligações diretas para os objetivos. Quanto aos interesses, não foram encontradas divergências em relação a teoria, visto que as expectativas de resultado e a autoeficácia contribuíram para a previsão dos interesses e, em conjunto com estes, ajudaram a explicar a variação nos objetivos de escolha em ambas as versões.

Verifica-se que a Teoria Sociocognitiva do Desenvolvimento de Carreira (Lent et al., 1994; Lent et al., 2004) propõe hipóteses para as diversas relações existentes ao longo do desenvolvimento profissional. Ao mesmo tempo, por ter sido elaborada de certo modo recentemente, estudos empíricos estão a contribuir para o refinamento de suas proposições, principalmente no que se refere à configuração das variáveis envolvidas na escolha de objetivos profissionais (Sheu et al., 2010). Com relação às asserções sobre o processo de desenvolvimento dos interesses, estas incitam a investigação da variável em diferentes contextos e populações, e os resultados das pesquisas já realizadas são bastante consistentes.

\section{A Teoria Desenvolvimentista de Donald Super}

$\mathrm{Na}$ teoria desenvolvimentista de Donald Super, os interesses fazem parte dos determinantes pessoais da tomada de decisão profissional ao lado de necessidades e valores, atitudes, desempenho, aptidões e hereditariedade (Super, 1957). Segundo o autor, os interesses podem ser caracterizados como positivos ou negativos em relação a situações ocupacionais (Super \& Bohn, 1970/1980), bem como indicam atividades e objetos específicos por meio dos quais os valores são alcançados (Super, 1973).

Existe uma natureza hierárquica na direção de necessidades, traços, valores e interesses. Necessidade é o conceito mais abrangente e, ao mesmo tempo, menos consciente, indica a carência de algo como amor e abrigo ou o desejo de se sentir realizado e de fazer algo construtivo. Os traços referem-se à forma de agir para satisfazer as necessidades em uma determinada situação. Necessidades e traços ajudam a compreender o estilo comportamental do indivíduo, mas para predizer comportamento educacional ou ocupacional é preciso traduzir as necessidades em objetivos operacionais. Assim, os valores são objetivos abstratos (p.ex., altruísmo, prestígio e retorno financeiro) implícitos no comportamento com a finalidade de satisfazer as necessidades, podem ser concretizados por meio de diferentes tipos de atividades ou objetos específicos (p.ex., trabalho social, ciência, negócios), o que se denomina interesses (Super, 1973).

São propostas por Super (1957; Super \& Bohn, 1970/1980) quatro definições de interesses, baseadas no método pelo qual eles são avaliados. O modo mais antigo de avaliar, denominado 'interesses expressos', se refere às declarações que o próprio indivíduo faz, informando sobre sua preferência por determinada profissão. Os 'interesses inventariados' são aqueles investigados por meio de inventários, que a partir de fundamentos estatísticos resumem as respostas das pessoas a um escore referente a grupos de profissões ou a tipos de atividades ocupacionais. Os 'interesses manifestos' são verificados a partir da comparação entre o que a pessoa diz se in- 
teressar e as atividades que ela realmente pratica. Por fim, os 'interesses testados' dizem respeito à avaliação dos conhecimentos que o indivíduo tem sobre determinado campo, uma vez que o aprendizado depende do interesse do sujeito.

Segundo o autor, o estudo sobre os interesses testados não atraiu a atenção dos pesquisadores em geral. Os interesses expressos ou preferência não são muito confiáveis quando avaliados na infância e início da adolescência, pois pessoas dessa faixa etária pensam em profissões mais inusitadas, divulgadas pela mídia, como peão de rodeio ou piloto. A partir da segunda metade da adolescência, os interesses expressos tendem a ser mais realistas e embora possuam alguma relação com os interesses inventariados, ainda têm pouca ligação com outros fatores que compõem a escolha profissional, como habilidades e valores. Os interesses manifestos, sejam eles hobbies ou atividades extraescolares, relacionam-se fracamente com a profissão escolhida, tais atividades geralmente são desenvolvidas para manter os relacionamentos de amizade ou têm associação com a fase em que estão vivendo, pouco se aproximam dos resultados de testes de interesses. Os interesses inventariados tendem a ser estáveis, enquanto as preferências podem mudar algumas vezes, por isso, possuem uma relação moderada. Porém, a escolha ou o ingresso na profissão em si tende a estar de acordo com os interesses inventariados, claro, desde que a pessoa tenha condições para investir no campo desejado (Super, 1957).

É importante reiterar que o conceito de interesses está inserido em uma teoria mais ampla acerca do desenvolvimento vocacional proposta por Super, sobre a qual os primeiros escritos datam de 1953. De acordo com o autor, o desenvolvimento vocacional é um processo que ocorre ao longo de toda a vida, por meio da implementação de autoconceitos (Super, 1953). O autoconceito é a representação de si mesmo em diferentes papeis, situação ou posição, no qual se desempenha uma série de funções e engendra determinadas relações. $\mathrm{O}$ autoconceito vocacional se refere ao papel que o indivíduo almeja exercer no trabalho, sendo formado por meio das experiências e modelos, bem como composto por vari- áveis pessoais, como os interesses. O grau de satisfação no trabalho é proporcional ao quanto se conseguiu implementar o autoconceito (Super, 1953; Super \& Bohn, 1970/1980). Para explicar esse movimento, Super foi construindo sua teoria, inicialmente, por meio de estudos empíricos iniciados na década de 1950, quando compreendia o processo de desenvolvimento vocacional a partir de uma sequência de estágios, que poderiam coincidir com determinadas faixas etárias, a saber, Crescimento (até 14 anos), Exploração (15 a 24 anos), Estabelecimento (25 a 44 anos), Manutenção (45 a 64 anos) e Desengajamento (a partir dos 65 anos; Super et al., 1954; Super \& Bohn, 1970/1980). Em 1980, Super apresenta uma atualização de sua teoria, a qual denominou de perspectiva life-span, life-space. Dessa forma, os estágios de desenvolvimento vocacional ocorrem longitudinalmente dentro de um maxiciclo (life-span), mas também podem acontecer várias vezes ao longo da vida, configurando-se como miniciclos, sempre que haja necessidade de realizar novas escolhas profissionais ou, até mesmo, pessoais, visto que nessa abordagem a carreira não se refere apenas ao desenvolvimento profissional, mas ao conjunto de papeis desempenhados ao longo da vida (life-space).

Ressalta-se que a maior parte dos textos que trata com profundidade dos interesses profissionais foi publicada anteriormente à revisão da teoria, uma vez que, a reformulação não incidiu sobre a função destes dentro do desenvolvimento de carreira. Em uma perspectiva longitudinal ou life-span, tem-se que os interesses começam a se definir na adolescência, tendendo a permanecerem estáveis após essa fase, assim, os estágios de vida importantes aqui são o de Crescimento e, principalmente, de Exploração (Super, 1954; Super \& Bohn, 1970/1980; Super et al., 1954).

Durante o estágio de Crescimento, a criança tende a fazer escolhas fantasiosas, os interesses estão sendo despertados e o reconhecimento das próprias capacidades ainda está no início. A formação do autoconceito se dá pela identificação com pessoas significativas da família e da escola. Nessa fase, as tarefas de desenvolvimento são: adquirir progressivamente autonomia em relação ao próprio comportamento; tornar-se 
consciente da importância das realizações escolares e profissionais; e adquirir as primeiras habilidades e atitudes de trabalho (Super \& Bohn, 1970/1980; Super et al., 1954).

No decorrer da adolescência e início da idade adulta, o indivíduo passa pelo estágio de Exploração. Esse momento é caracterizado pela busca de autoconhecimento e informações profissionais, inclusive por meio de tentativas e experimentação de papeis (Super \& Bohn, 1970/1980; Super et al., 1954). Essa etapa envolve três tarefas, quais sejam, cristalização, especificação e implementação de uma preferência ocupacional. A cristalização, mais especificamente, a cristalização dos interesses profissionais, ocorre entre 14 e 18 anos. A tarefa consiste em formular uma preferência geral, que se expresse de forma constante. Essa tarefa pode ser facilitada, ainda, quando há sucesso nas etapas do estágio de Crescimento. Entretanto, para que consiga realizá-la, o adolescente deve estar ciente da necessidade da tarefa, levando em consideração seus aspectos pessoais (p.ex., capacidades e habilidades) e as informações advindas do ambiente, por exemplo, por meio dos pais e professores. Quanto mais definido for seu conhecimento em relação aos interesses, mais diferenciado será seu perfil e mais fácil será a tarefa de cristalizar uma preferência. Tendo definido uma preferência geral e concluído a exploração de ocupações, é possível especificar uma determinada carreira a ser seguida. Isso ocorre, geralmente, entre 18 e 21 anos. Posteriormente, o jovem vai implementar sua escolha, ou seja, vai ingressar em um trabalho ou sistema educacional especializado para vivenciar o autoconceito na carreira escolhida (Savickas, 2002; Super, 1957).

Os interesses estão relacionados ao campo e ao nível de uma ocupação, por exemplo, no campo da engenharia mecânica existem ocupações de diferentes níveis profissionais, como o engenheiro, o mecânico, o operador de máquinas. Assim, uma pessoa com interesse em engenharia pode não ter recursos e oportunidades para se tornar um engenheiro e, então, seguir a profissão de mecânico (Super et al., 1954). Nesse sentido, os interesses são responsáveis pela direção e persistência dentro de uma área ocupacional, mas, aparentemente, não é determinante da quantidade de esforço dentro dela. De modo que quanto mais os interesses estiverem definidos e compatíveis com as atividades desenvolvidas, maior será a satisfação do indivíduo no trabalho (Super, 1957; Super \& Bohn, 1970/1980).

Super é um dos precursores da abordagem desenvolvimental da escolha profissional e sua teoria é amplamente utilizada em contextos de orientação profissional e de carreira. Nota-se que esse teórico é preocupado não apenas com a investigação e construções teóricas, mas também com a aplicação prática de sua abordagem (Super, 1983). Tal perspectiva vem sendo desenvolvida ao longo dos anos, expandindo seus domínios para outros construtos, além dos interesses, também relevantes para a área de orientação profissional e de carreira (Savickas, 2005).

\section{A Teoria Construtivista de Mark Savickas}

Para finalizar a exposição sobre as teorias que explanam acerca dos interesses profissionais e a relevância de sua avaliação, apresentam-se as concepções de Savickas (1995, 1999, 2002, 2005). Esse autor ampliou a perspectiva de Super (1980), estabelecendo conexões com uma abordagem construtivista (Savickas, 2002, 2005), na qual para compreender o desenvolvimento de carreira não é necessário apenas conhecer os processos de escolha e experiências ocupacionais, mas o contexto em que ocorrem e o significado que o indivíduo atribui a suas vivências na busca pela adaptação ao ambiente em que vive. Assim, para Savickas (1995), os interesses denotam uma interação entre o indivíduo e o ambiente. De modo objetivo, a avaliação dos interesses indica padrões de gosto, rejeição e indiferença ideia compartilhada por autores como Lent et al. (1994) -, e subjetivamente consiste em forma de expressão da personalidade - o que é consoante com a perspectiva teórica de Holland (1997).

Como expressão da personalidade, os interesses fazem parte de uma matriz motivacional que inclui as necessidades e os valores (Savickas, 1995). Essas três variáveis podem ajudar a explicar o movimento do indivíduo no mundo ao responder a três questões 'por 
que', 'o quê' e 'como' o indivíduo se move. As necessidades (por que) indicam aquilo que falta no indivíduo para que ele se sinta mais seguro e completo, impulsionam e geram energia para o movimento. Os valores (o quê) correspondem aos objetos ou recompensas que podem satisfazer as necessidades, ou seja, eles ditam a direção do movimento. Os interesses (como) são a ponte pela qual as necessidades e os valores se conectam, modulam o movimento. Por exemplo, algumas pessoas expressam necessidades tanto de ajudar quanto de controlar os outros. Isto os leva ao altruísmo (valor) e, finalmente, a desenvolver interesses em ocupações de cunho social, como ensino, aconselhamento e assistência social. Tais profissões permitem aos seus ocupantes fornecer aos clientes conselhos úteis, assim satisfazendo as necessidades de amparo e controle. Notase, nessa definição, grande similaridade às concepções de Super (1973) quando se refere à hierarquia entre necessidade e interesses.

Os interesses podem, também, serem traduzidos como soluções que o indivíduo dá para problemas pessoais, para algo que lhe faltou no decorrer da existência. Os problemas se originam no contexto familiar e depois são transportados para o ambiente social. A solução pode surgir de modelos que se sobressaem em determinada cultura como forma de atingir a autorrealização naquela sociedade. A partir da narrativa e dos inventários é possível identificar os materiais e recursos que conduzem à autorrealização e completam o projeto de vida. Enquanto estilo do comportamento, os interesses dependem do ambiente disponível e da estrutura de oportunidades. Assim, ao compará-los a outros construtos como necessidades e valores, eles possuem menor estabilidade (Savickas, 1995).

Posteriormente, na busca por uma definição que contemplasse todas as dimensões do conceito de interesses, Savickas (1999) faz uma revisão das principais perspectivas teóricas que abordam o construto. Conclui que, subjacentes aos interesses, estão processos cognitivos, emocionais, volitivos e comportamentais. Entretanto, o autor não encontrou uma teoria que unificasse o conceito, pois cada abordagem descreveu o construto por meio de uma propriedade específica.
Assim, com o intuito de especificar o que é interesse, o autor o definiu em duas formas, a saber, interesse como estado e como traço.

$\mathrm{O}$ interesse como um estado de consciência é caracterizado por uma prontidão para pensar ou responder a um estímulo específico do ambiente, como objetos, atividades, pessoas e experiências. Ao identificar um estímulo, o sujeito dirige sua atenção para ele, antecipa sentimentos agradáveis e realiza uma avaliação favorável. Essa avaliação gera o desejo (volição) de fazer algo em relação ao estímulo (p.ex., aprender mais sobre ele) devido à expectativa de satisfação ou gratificação. Tal antecipação de resultado conduz o indivíduo a um curso de ação em direção ao objeto, com a finalidade de satisfazer desejos, necessidades ou valores pessoais. Caso o indivíduo realmente se identifique com a atividade, poderá incorporá-la como um novo interesse ao sistema de autoconceito existente, sendo que a representação simbólica desse interesse se dá pelo estímulo que evoca atenção e ação (p.ex., eu gosto de livros). Dessa forma, o interesse é um esforço adaptativo para usar o ambiente a favor das necessidades e valores.

Enquanto traço, o interesse se refere a uma disposição geral para responder a um grupo homogêneo de estímulos específicos, configurando-se em interesses. Assim, a forma singular - interesse - que denota um estado ou ação passa, para o plural - interesses - caracterizando um conceito ou traço. Por traço, entende-se uma tendência de resposta disposicional, consistente e estável, a um determinado grupo de estímulos ambientais. Por meio de análises do comportamento e autobiografias, é possível ver a facilidade e frequência com a qual um interesse é iniciado, bem como sua duração ao longo do tempo. Já por meio de inventários de interesses, é possível definir a força relativa, ou seja, o quanto uma preferência por determinada atividade se sobressai quando comparada a outros interesses. Ter consciência dessa disposição conduz à localização de um código no mundo do trabalho e ao desenvolvimento do sistema de autoconceitos existente. A autoconsciência dessa representação promove a estabilidade e a continuidade da disposição. 
Segundo Savickas (1999), quanto aos fatores que contribuem para a origem e desenvolvimento dos interesses, tem-se a hereditariedade, experiências de aprendizagem, percepção de capacidade, identificação com modelos, acomodação de papeis sociais, expressão da personalidade e implementação de autoconceito. $\mathrm{O}$ autor reuniu e apresentou estudos e teorias sobre esses aspectos de forma expositiva, sem delimitar preferências ou conclusões que ultrapassassem as descrições.

Nota-se que Savickas $(1995,1999)$ debruçou-se sobre a tarefa de delimitar o que são interesses profissionais, mesmo tendo atualmente uma teoria bem mais abrangente que trata de outros aspectos do desenvolvimento ocupacional (Savickas, 2005; Savickas \& Porfeli, 2012). Sua revisão e conclusão a respeito do significado do termo permitem a compreensão e diferenciação de outros construtos. Ao mesmo tempo, sua proposta de conceituação possui consistência teórica e prática que possibilita a investigação dos interesses enquanto construto psicológico (Leitão \& Miguel, 2001).

\section{Conclusões}

Uma vez que os inventários de interesses profissionais são vitais para o campo de desenvolvimento de carreira (Savickas, 2013), o presente estudo se faz útil aos pesquisadores e profissionais da área ao compilar conceitos sobre os interesses que advém de teorias que sugerem esse tipo de avaliação. Ultrapassando o limite descritivo, pretende-se neste momento apresentar uma conclusão a respeito do construto tratado e indicar futuras pesquisas que possam dar continuidade ao seu desenvolvimento teórico e aplicação prática.

Assim como observado por outros estudiosos (Leitão \& Miguel, 2001), nota-se que a definição proposta por Savickas (1999) parece a mais apropriada, pois conseguiu integrar as concepções existentes e ao mesmo tempo delimitar o construto. Sendo que a ideia de interesses profissionais como um traço, tendência de resposta relativamente estável a um grupo de estímulos, tem sido amplamente aceita (Sodano, 2015). Possivelmente, esse é o conceito mais adequado porque vem de uma elaboração construída a partir da revisão teórica de proposições anteriores, enquanto as demais se fundamentaram, principalmente, na experiência prática, na construção de inventários ou em componentes isolados.

Quanto às teorias de Holland (1997), Lent et al. (1994) e Super (1954, 1957), embora não se tenha um consenso sobre o conceito de interesses, verifica-se que não há acentuadas divergências ou conflitos. Ao reunir as definições, é possível concordar com Hansen (2005) que, em sua revisitação aos conceitos de interesses, visualiza o construto como reflexo de três componentes, a saber, personalidade, motivação/direção e autoconceito/identificação. De modo geral, os teóricos aqui apresentados se referem aos interesses profissionais como uma especificação de preferências, que tende a estabilidade e, que pode conduzir o indivíduo a buscar determinadas situações e elementos ocupacionais.

Quanto ao desenvolvimento dos interesses profissionais, notam-se diferenças entre as teorias no que se refere, principalmente, à ênfase nas experiências e nos aspectos socioeconômicos e culturais. De fato, algumas teorias sobre carreira focam mais no conteúdo, ou seja, elementos que compõe o desenvolvimento de carreira; outras focam mais nos processos, isto é, no desenvolvimento e interação dos elementos; e ainda há aquelas que abordam conteúdos e processos (Patton \& McMahon, 2014).

Por exemplo, as proposições de Holland (1997) quanto ao desenvolvimento dos interesses, incluem, mas não aprofundam a explanação sobre os fatores que contribuem para a formação das personalidades vocacionais. Essa teoria não se propõe a fornecer explicações desenvolvimentais, mas sim, hipóteses de como os interesses em seu estado atual se manifestam no ambiente de trabalho. Super $(1954,1957,1980)$, por sua vez, limita o desenvolvimento dos interesses às relações com pessoas significativas, autoconhecimento e busca de informações, tendo o contexto socioeconômico participação explícita apenas no momento de formulação de objetivos profissionais. 
Retomando as observações de Leitão e Miguel (2001), verifica-se, também, que a abordagem de Savickas (1999) não aprofunda as relações existentes ao longo do desenvolvimento dos interesses, sendo a teoria sociocognitiva (Lent et al., 1994; Lent et al., 2004) a que mais esclarece sobre as associações com fatores pessoais e contextuais, sobretudo no que diz respeito ao papel das experiências na modulação dos interesses (Hansen, 2005; Leitão \& Miguel, 2001; Patton \& McMahon, 2014). Novamente, cabe destacar aqui as proposições de Hansen (2005) que, ao buscar na literatura elementos sobre o desenvolvimento dos interesses, sintetizou as informações em dois componentes. $\mathrm{O}$ primeiro está relacionado à criação, referindose aos fatores de socialização e aprendizagem, incluindo também influências ambientais e psicológicas que moldam os interesses. O segundo, chamado natureza, diz respeito à genética e hereditariedade.

Em relação à avaliação em processos de orientação profissional e de carreira, percebe-se que os autores aqui focalizados são unânimes em destacar a relevância dos interesses, havendo entre eles algumas diferenças quanto à utilização de outras variáveis no processo de orientação. Holland (1997), por exemplo, ao compreender os interesses como expressão da personalidade, propõe um meio de avaliação que engloba aspectos como competências e habilidades, enquanto, Savickas (1999) e Super (1983) destacam que as habilidades e os valores devem ser analisados concomitantemente, e Lent et al. (1994; Lent et al., 2004) indicam as crenças de autoeficácia e as expectativas de resultado. De modo geral, os teóricos consideram que os interesses profissionais estão diretamente relacionados aos objetivos de carreira, sendo que as pesquisas empíricas realizadas confirmam esse pressuposto, bem como sua consistência ou estabilidade ao longo do tempo (Hansen, 2005; Rounds \& Su, 2014; Sodano; 2015).

Em síntese, as perspectivas teóricas aqui reunidas, embora diferentes, podem ser complementares. Percebe-se, inclusive, que os autores citam uns aos outros, sugerindo seus instrumentos ou indicando as contribuições de uma determinada concepção. Portanto, o objetivo deste artigo se encerra à medida que foi possível conhecer as proposições de alguns teóricos que dissertaram sobre o desenvolvimento dos interesses profissionais e que contribuíram para a sua evolução conceitual.

A título de agenda de pesquisa, são indicadas mais investigações, inclusive longitudinais, no contexto nacional que tenham como finalidade (a) avaliar como ocorre o desenvolvimento dos interesses, (b) de que forma as variáveis contribuem para esse processo, e (c) como sua avaliação pode ser aprimorada de modo a instrumentalizar não apenas aqueles profissionais que trabalham diretamente com orientação profissional, mas também, educadores e gestores.

O último ponto deve ser tratado com urgência, uma vez que, no contexto brasileiro, há apenas cinco testes psicológicos que avaliam interesses aprovados para o uso profissional do psicólogo (Sistema de Avaliação de Testes Psicológicos [SATEPSI], 2016). Entretanto, somente dois inventários (Bandeira \& Levenfus, 2009; Noronha, Sisto, \& Santos, 2007) e um teste de autoexpressão encontram-se disponíveis para compra (Jacquemin, 2000; Jacquemin, Okino, Noce, Assoni, \& Pasian, 2006). Outros recursos de avaliação são três técnicas de natureza visual (jogo com cartões; Coelho, 2015; Magalhães, 2011; Neiva, 2015). Portanto, são necessárias a ampliação do número e a diversificação dos instrumentos de interesses, pois permitiriam que diferentes populações se beneficiassem desse conhecimento, aqueles que buscam orientação nos momentos de escolha, e também, os que frequentam ambientes acadêmicos e profissionais e não recorrem a um profissional especializado quando vivenciam tomadas de decisões de carreira.

\section{Referências}

Ambiel, R. A. M., \& Noronha, A. P. P. (2014). Escala de Autoeficácia para Escolha Profissional: Manual técnico (2. ed.). São Paulo, SP: Casa do Psicólogo.

Bandeira, D. R., \& Levenfus, R. S. (2009). Avaliação dos Interesses Profissionais - AIP. São Paulo, SP: Vetor. 
Bandura, A. (1977). Self-efficacy: Toward a unifying Theory of Behavioral Change. Psychological Review, 84(2), 191-215. doi:10.1037/0033295X.84.2.191

Bandura, A. (1982). Self-efficacy mechanism in human agency. American Psychologist, 37(2), 122-147. doi:0003-066x/82/3702-0122800.75

Bandura, A. (2008). A evolução da teoria social cognitiva. In A. Bandura, R. G. Azzi, \& S. Polydoro (Eds.), Teoria Social Cognitiva: Conceitos Básicos (pp. 15-42). Porto Alegre, RS: Artmed. (Original publicado em 1978)

Byars-Winston, A., Estrada, Y., Howard, C., Davis, D., \& Zalapa, J. (2010). Influence of social cognitive and ethnic variables on academic goals of underrepresented students in science and engineering: A multiple-groups analysis. Journal of Counseling Psychology, 57(2), 205-218. doi:10.1037/a0018608

Coelho, M. E. (2015). Conhecendo as profissões com a técnica R.O.. São Paulo, SP: Hogrefe Centro Editor de Testes e Pesquisas em Psicologia.

Fryer, D. H. (1931). The measurement of interests. New York: Holt.

Hansen, J. C. (2005). Assessment of interests. In S. D. Brown \& R. W. Lent (Eds.), Career development and counseling: Putting theory and research to work (pp. 281-304). Hoboken, NJ: John Wiley \& Sons.

Hirschi, A. (2010). Individual predictors of adolescents' vocational interest stabilities. International Journal for Educational and Vocational Guidance, 10(1), 5-19. doi:10.1007/s10775-0099171-2

Hirschi, A. (2011). Relation of vocational identity statuses to interest structure among Swiss adolescents. Journal of Career Development, 38(5), 390-407. doi:10.1177/0894845310378665

Holland, J. L. (1959). A theory of vocational choice. Journal of Counseling Psychology, 6(1), 35-45. doi:10.1037/h0040767

Holland, J. L. (1996). Exploring careers with a typology: What we have learned and some new directions. American Psychologist, 51(4), $397-$ 406. doi:10.1037/0003-066X.51.4.397

Holland, J. L. (1997). Making vocational choices: A theory of vocational personalities and work environments (3. ed.). Odessa, FL: Psychological Assessment Resources.
Harrington, T., \& Long, J. (2013). The history of interest inventories and career assessments in career counseling. The Career Development Quarterly, 61, 83-92. doi:10.1002/j.21610045.2013.00039.x

Huang, J. L., \& Pearce, M. (2013). The other side of the coin: Vocational interests, interest differentiation and annual income at the occupation level of analysis. Journal of Vocational Behavior, 83(3), 315-326. doi:10.1016/j.jvb.2013.06.003

Jacquemin, A. (2000). O BBT-Br: Teste de Fotos de Profissões: Normas, adaptação brasileira, estudos de caso. São Paulo, SP: Centro Editor de Testes e Pesquisas em Psicologia.

Jacquemin, A., Okino, E. T. K., Noce, M. A., Assoni, R. F., \& Pasian, S. R. (2006). O BBTBr Feminino. Teste de Fotos de Profissões: Adaptação brasileira, normas e estudos de caso. São Paulo, SP: Centro Editor de Testes e Pesquisas em Psicologia.

Leitão, L. M., \& Miguel, J. P. (2001). Os interesses revisitados. Psychologica, 26, 79-104. Recuperado em http://staff.fpce.uc.pt//jpacheco/pdf/ Psychologica26_79_104.pdf

Lent, R. W., \& Brown, S. D. (2006a). On conceptualizing and assessing social cognitive constructs in career research: a measurement guide. Journal of Career Assessment, 14(1), 12-35. doi:10.1177/1069072705281364

Lent, R. W., \& Brown, S. D. (2006b). Integrating person and situation perspectives on work satisfaction: A social-cognitive view. Journal of Vocational Behavior, 69, 236-247. doi:10.1016/j. jvb.2006.02.006

Lent, R. W., \& Brown, S. D. (2008). Social cognitive career theory and subjective well-being in the context of work. Journal of Career Assessment, 16, 6-21. doi:10.1177/1069072707305769

Lent, R. W., \& Brown, S. D. (2013). Social cognitive model of career self-management: toward a unifying view of Adaptive Career Behavior Across the Life Span. Journal of Counseling Psychology, 60(4), 557-568. doi:10.1037/ a0033446

Lent, R. W., Brown, S. D., \& Hackett, G. (1994). Toward a unifying social cognitive theory of career and academic interest, choice and performance. Journal of Vocational Behavior, 45, 79-122. doi:10.1006/jvbe.1994.1027 
Lent, R., Hackett, G., \& Brown, S. D. (2004). Una perspectiva social cognitiva de la transición entre la escuela y el trabajo. Evaluar, 4, 1-22. Recuperado em http://revistas.unc.edu.ar/index. $\mathrm{php} /$ revaluar/article/view/596/565

Magalhães, M. O. (2011). Matriz de habilidades $e$ interesses profissionais. São Paulo, SP: Casa do Psicólogo.

Mattiazzi, B. (1977). A natureza dos interesses e a orientação vocacional (3. ed.). Petrópolis, RJ: Vozes.

Nauta, M. M. (2010). The development, evolution, and status of Holland's theory of vocational personalities: Reflections and future directions for counseling psychology. Journal of Counseling Psychology, 57(1), 11-22. doi:10.1037/ a0018213

Neiva, K. M. C. (2015). Critérios para escolhas profissionais (3. ed.). São Paulo, SP: Vetor

Noronha, A. P., Sisto, F., \& Santos, A. A. A. (2007). Escala de Aconselhamento Profissional -EAP Manual Técnico (Brasil). São Paulo, SP: Vetor.

Nunes, M. F. O. (2007). Escala de Fontes de Eficácia Percebida: Aplicação com jovens em escolha profissional (Dissertação de mestrado, Programa de Pós-Graduação Stricto Sensu em Psicologia, Universidade São Francisco, Itatiba, SP, Brasil).

Nunes, M. F. O. L. (2009). Estudos psicométricos da escala de autoeficácia para atividades ocupacionais (Tese de Doutorado, Programa de Pós-Graduação Stricto Sensu em Psicologia, Universidade São Francisco, Itatiba, SP, Brasil).

Nunes, M. F. O., \& Noronha, A. P. P. (2009). Autoeficácia para atividades ocupacionais e interesses profissionais em estudantes do ensino médio. Psicologia: Ciência e Profissão, 29(1), 102-115. Recuperado em http://www.scielo.br/pdf/pcp/ v29n1/v29n1a09.pdf

Nunes, M. F. O., \& Noronha, A. P. P. (2011). Associações entre auto-eficácia para atividades ocupacionais e interesses em adolescentes. Psicologia: Reflexão e Crítica, 24(1), 1-9. doi:10.1590/S0102-79722011000100002

Pajares, F., \& Olaz, F. (2008). Teoria Social Cognitiva e autoeficácia: Uma visão geral. In A. Bandura, R. G. Azzi, \& S. Polydoro (Eds.), Teoria Social Cognitiva: Conceitos Básicos (pp. 97-114). Porto Alegre, RS: Artmed.
Patton, W., \& McMahon, M. (2014). Career Developmentand Systems Theory: Connecting theory and practice ( $3^{\text {rd }}$ ed.). Rotterdam, The Netherlands: Sense.

Primi, R., Muniz, M., Mansão, C. M., \& Nunes, M. F. O. (2010). SDS - Questionário de busca auto-dirigida - Manual técnico da versão brasileira. São Paulo, SP: Casa do Psicólogo.

Rounds, J., \& Su, R. (2014). The nature and power of interests. Current Directions in Psychological Science, 23(2), 98-103. doi:10.1177/0963721414522812

Savickas, M. L. (1995). Examining the personal meaning of inventoried interests during career counseling. Journal of Career Assessment, 3(2), 188-201. doi:10.1177/106907279500300206

Savickas, M. L. (1999). The Psychology of Interests. In M. L Savickas \& A. R. Spokane (Eds.), Vocational interests: Meaning, measurement and counseling use (pp. 19-56). Palo Alto, CA: Davies-Black.

Savickas, M. L. (2002). Career construction: A developmental theory of vocational behavior. In D. Brown (Ed.), Career choice and development ( $4^{\text {th }}$ ed., pp. 149-205). San Francisco, CA: Jossey-Bass.

Savickas, M. L. (2005). The Theory and Practice of Career Construction. In S. D. Brown \& R. W. Lent (Eds.), Career development and counseling: Putting theory and research to work (pp. 42-70). Hoboken, NJ: John Wiley \& Sons.

Savickas, M. L. (2013). Ten Ideas that Changed Career Development. In M. L. Savickas (Ed.), Ten Ideas that Changed Career Development (pp. 1-3). Broken Arrow, OK: National Career Development Association.

Savickas, M. L., \& Porfeli, E. J. (2012). Career Adapt-Abilities Scale: Construction, reliability, and measurement equivalence across 13 countries. Journal of Vocational Behavior, 80(3), 661-673. doi:10.1016/j.jvb.2012.01.011

Sheu, H. B., Lent, R. W., Brown, S. D., Miller, M. J., Hennessy, K. D., \& Duffy, R. D. (2010). Testing the choice model of social cognitive career theory across Holland themes: A meta-analytic path analysis. Journal of Vocational Behavior, 76(1), 252-264. doi:10.1016/j.jvb.2009.10.015

Sistema de Avaliação de Testes Psicológicos. (2016). Lista completa dos testes. Recuperado em http:// satepsi.cfp.org.br/listaTesteFavoravel.cfm 
Sodano, S. M. (2015). Meaning, measurement, and assessment of vocational interests for career intervention. In P. J. Hartung, M. L. Savickas, \& W. B. Walsh (Eds.), APA Handbook of Career Intervention: Volume 1. Foundations (pp. 281301). Washington, DC: American Psychological Association

Super, D. E. (1953). A theory of vocational development. American Psychologist, 8(5), 185-190. doi:10.1037/h0056046

Super, D. E. (1954). The measurement of interests. Journal of Counseling Psychology, 1(3), 168172. doi: $10.1037 / \mathrm{h} 0059084$

Super, D. E. (1957). The Psychology of Careers. New York: Harper and Row.

Super, D. E. (1973). The work values inventory. In D. G. Zytowski (Ed.), Contemporary approaches to interest measurement (pp.189-205). Minneapolis, MN: University of Minnesota Press.

Super, D. E. (1980). A life-time, life-space, approach to career development. Journal of Vocational Behavior, 16, 282-298. doi:10.1016/00018791(80)90056-1

Super, D. E. (1983). Assessment in career guidance: Toward truly developmental counseling. Personnel and Guidance Journal, 61(9), 555-562. doi:10.1111/j.2164-4918.1983.tb00099.x

Super, D. E., \& Bohn, M. J., Jr. (1980). Psicologia Ocupacional (E. Nascimento \& F. Santos, Trads.). São Paulo, SP: Atlas. (Original publicado em 1970)
Super, D. E., Crites, J. O., Hummel, R. C., Moser, H. P., Overstreet, P. L., \& Warnath, C. F. (1954). Vocational development: a framework for research. New York: Columbia University.

Tracey, T. J. G. (2010). Relation of interest and selfefficacy occupational congruenceand career choice certainty. Journal of Vocational Behavior, 76, 441-447. doi:10.1016/j.jvb.2009.10.013

Tracey, T. J. G., Wille, B., Durr, M. R., II, \& De Fruyt, F. (2014). An enhanced examination of Holland's consistency and differentiation hypotheses. Journal of Vocational Behavior, 84, 237-247. doi:10.1016/j.jvb.2014.01.008

Werlang, B. S. G., Villemor-Amaral, A. E., \& Nascimento, R. S. G. F. (2010). Avaliação psicológica, testes e possibilidades de uso. In Conselho Federal de Psicologia (Ed.), Avaliação psicológica: Diretrizes na regulamentação da profissão (pp. 101-128). Brasília, DF: Conselho Federal de Psicologia.
Recebido: 06/01/2016

$1^{a}$ revisão: $17 / 04 / 2016$ Aceite final: 26/04/2016 